\title{
Longitudinal Study of Looming in Infants with High-Density EEG
}

\author{
Audrey L.H. van der Meer Monica Svantesson F.R. Ruud van der Weel \\ Developmental Neuroscience Laboratory, Department of Psychology, Norwegian University of Science and \\ Technology, Trondheim, Norway
}

\section{Key Words}

Perceptual development $\cdot$ Timing $\cdot$ Time-to-collision • Infant brain • Visual looming $\cdot$ Visual evoked potential $\cdot$ Prospective control $\cdot$ Role of locomotor experience · Motion perception

\begin{abstract}
A rapidly approaching object provides information about the object's approach and how imminent a collision is. Prospective control when responding to a looming virtual object approaching on a direct collision course was studied longitudinally in 10 infants aged 5/6 and 12/13 months. Different characteristics of the looming-related visual evoked potential (VEP) responses from infants' brain electrical recordings (EEG) were explored and compared between the infants at these different ages. The aim of this study was to find evidence for infant brain electrical responses coherent with a looming stimulus approaching the infant under three different accelerations. It was also investigated whether the use of different timing strategies to estimate the loom's time-to-collision would produce differences in the EEG recordings. The results showed that the timing and the duration of the VEP responses differed with age. At the age of 5/6 months, infants showed VEP peaks earlier in the looming se-
\end{abstract}

quence and VEP responses with longer duration than when they were 12/13 months old. Results from the timing-strategy analysis showed that with age, 4 infants shifted from a less efficient timing strategy involving the loom's velocity to the more efficient strategy involving the loom's time-to-collision. Further, it was found that peak VEP activation in the investigated areas propagated across the cortex, showing the highest observed activation in the occipital area at the age of $5 / 6$ months, whereas the parietal area showed the highest activation when the infants were $12 / 13$ months. The decrease in processing time together with a peak VEP response closer to the loom's time-to-collision indicate a developmental trend in infants' prediction of an object's timeto-collision. This developmental trend is further substantiated by the shift from a less efficient to a more efficient timing strategy and by evidence of propagated peak VEP activation towards higher information processing areas in the visual pathway with age. As infants grow older and become more mobile, one of the underlying causes of the developmental trend found in our study could be due to an increase in locomotor experience. More follow-up research is needed to investigate the relation between behavioural development and changes in brain activity associated with infants' perception of looming motion.

Copyright $\odot 2012$ S. Karger AG, Basel

\section{KARGER}

Fax +41613061234

E-Mail karger@karger.ch

www.karger.com
(C) 2012 S. Karger AG, Basel

$0378-5866 / 12 / 0346-0488 \$ 38.00 / 0$

Accessible online at:

www.karger.com/dne
Audrey L.H. van der Meer, $\mathrm{PhD}, \mathrm{MSc}$

Developmental Neuroscience Laboratory, Department of Psychology

Norwegian University of Science and Technology (NTNU)

NO-7491 Trondheim (Norway)

E-Mail audrey.meer@svt.ntnu.no 


\section{Introduction}

How does the infant brain process information of an imminent collision of a looming object? In order to reach our destination and to avoid objects in our path, changes of direction have to be made. Moving towards a specific place at a specific time when interacting with the environment involves being prepared to do specific things. This entails that all movements have to be controlled by perceiving what is likely to happen next. This is achieved by foreseeing ongoing events in our surroundings, as well as the unfolding of our own actions. Interacting with the environment demands highly efficient systems for integration of visual information around which adaptive behaviour develops [1]. Navigation and interaction with events in the surroundings demand prospective control where action, perception, and cognition are tightly coupled together in a complex system [2-4].

The information needed for the perception of our own motion or motion in the environment is available in the optical flow field [5]. Perception of movement in our visual field transforms the entire optic array into a flow field, which provides information of the direction of motion. Through development the expansion of prospective control has to do with the control over and above the information available to guide our actions [2-4]. One of the earliest indications of prospective behaviour seen in infants is the development of smooth visual pursuit of moving objects, and involves infants' anticipation of a moving object's forthcoming motion. This ability develops over the first 3 months of life $[3,6,7]$.

Studies performed on monkeys were the first attempts to analyse the functionally specialized areas of visual motion $[8,9]$. These studies point to a localization of visual processing in the middle temporal (MT) and medial superior temporal area (V5). The eyes' perception of visual information propagates from the lateral geniculate nucleus to the primary cortex. The information in the primary visual cortex is distinguished by two parallel interconnected pathways, either through a dorsal stream propagating information towards the parietal lobe or through a ventral stream directed towards the temporal lobe. The dorsal stream is thought to be involved in the processing of movement while the ventral stream is thought to be a part of the processing of object recognition [10-14]. A growing number of studies shows that the dorsal stream in the adult cortex and its incorporated areas (V1, V2, $\mathrm{MT}+$ ) have neurons that are selectively activated by motion stimuli [15-17]. The functioning and development of these areas for infants is less known.

Longitudinal Study of Looming in

Infants with High-Density EEG
Research on infant perception was at its peak in the 1960s and 1970s, studying how soon infants could respond to motion stimuli and describing characteristics that were being processed. Experimenters caught an interest in looming stimuli and investigated defensive responses [18-20]. The ability to detect and avoid dangerous situations is one of the most important abilities for all living creatures. Throughout the animal kingdom an essential function of the brain is to detect threats, and threats are often posed by objects or predators on a collision course. An object on a direct collision course will project an expanding image with a rapid symmetrical growth on the retina. This motion is referred to as looming and on a direct collision course it provides information about the approaching object and how imminent the collision is [19].

The notion that perception of visual motion represents an important ability of our visual system has brought a large number of studies trying to map the specialized processing of this type of stimuli. Neurons that react to such stimuli have been found in animal data [21-23] and in adult data [10], and there is ample evidence that the human visual system is specialized to detect and respond to looming motion [11-13].

There are several theories about the underlying causes of the development of the ability to process the available information in the surrounding world. Studies have reported that sensitivity to optic flow shows a significant increase after infants have started crawling [24-29], while other findings argue that selective responses are due to synaptic maturation and myelination $[30,31]$ of the visual cortical areas $[32,33]$.

The relationship between brain and behavioural development varies according to the theoretical framework that is used to highlight this relationship. Traditional frameworks base the relation between brain and behavioural development on brain maturation [34]. Traditional frameworks argue that particular regions of the brain have to mature in order for new motor, cognitive, or sensory functions to appear. The models presented from this view often relate changes in behaviour to the physical growth of the brain. An alternative framework for analysing and interpreting postnatal functional brain development in human infants has been presented where it is argued that an integration of information about structural brain development with knowledge about perceptual, cognitive, and behavioural changes is necessary in order to fully understand the relation between these components and how they interact [34]. According to such a framework, functional brain development can be ana-

Dev Neurosci 2012;34:488-501 
lysed in terms of the differential specialization of multiple co-active pathways, and differential specialization between and within pathways which occur during development.

Studies have investigated infants' development of prospective control and how they process information of looming motion by looking at infants' defensive responses to looming stimuli [18-20,35-37]. Today it is well established that blinking is the most reliable indicator of awareness to stimuli on a collision course in early infancy. In order to protect our eyes from something that is about to hit our face, the timing of the defensive blink needs to be precise. If the blinking occurs too early, there is a risk that the eyes are reopened even before the collision takes place, or the blinking may appear too late so that the eyes may get injured. The estimate of the response has to be made according to when an object is about to hit, referred to as the time-to-collision. This requires precise prospective control where the defensive action has to be perceived ahead in time so that the body is prepared to respond [2-4].

Recent behavioural studies [35-37] have investigated infants' defensive blinking in response to a virtual looming stimulus heading towards them. The stimuli were programmed to loom towards the infant and approach the infant's face so that the infant would get an experience of a visual collision. Characteristics of the defensive blinking behaviour were examined by looking at different timing strategies and which information variable the strategies were based on. Infants mainly timed their defensive blinking by relying on one of three information variables based on the stimulus' visual angle, velocity, or time away from the virtual collision. The results showed that infants initially used a strategy based on visual angle or velocity and switched to a strategy based on time around the age of 7 months. The results also showed that infants that relied on a strategy based on visual angle or velocity had a higher number of late defensive blinks than infants using a time strategy. This led the researchers to argue that the findings revealed a developmental trend where infants shifted from a less useful to a more sophisticated timing strategy that was less prone to misjudgements. This shift from a less useful variable to a more useful variable has also been found in adult data of perceptual judgments of a simulated collision [38, 39].

The use of high-density EEG allows the investigation of how the human brain processes information available in our surroundings. The advances in the technology of recording event-related electrical potentials at the scalp make it possible to use this method with young infants
[40]. In a recent study, infant brain electrical responses to a looming stimulus were recorded and it was investigated how the infant nervous system extracts and processes information for impending collision in a cross-sectional design [41]. The findings from this study indicate that looming-related brain activity in infants between 5 and 11 months of age is characterized by theta oscillations, and source analyses revealed that this activity was localized in the visual cortex. The results showed a clear developmental trend where it took the youngest infants (5-7 months) about twice as long to process the looming information and they were not able to differentiate between the loom's three different accelerations, whereas the eldest infants (10-11 months) displayed very short, tidy, and separated brain waves.

In the present study, high-density EEG measurements of infants' brain electrical activity are used to study infants' perception and processing of a looming virtual object that approaches them on a direct collision course. This method is used to detect electrical changes as groups of neurons fire within the cerebral cortex. The cerebral cortex is associated with higher cognitive functioning and is also thought to play a central role in the development of cognitive and perceptual development [40]. The aim of this study is to investigate whether evidence from recent behavioural studies of infants' prospective responses to looming stimuli can be confirmed in their looming-related brain electrical activity. Further, the development of prospective control of the timing of looming-related brain responses will be investigated through a longitudinal perspective by testing infants at the age of $5 / 6$ months and again at the age of 12/13 months. Characteristic features of infant brain responses will also be investigated from a developmental perspective by determining which timing strategies infants use to estimate the loom's time-to-collision as it approaches them under three different accelerations.

\section{Methods}

Participants

A total of 14 babies were recruited from newspaper birth announcements to take part in the experiment, but only 10 of these provided longitudinal data for the analyses. Three babies had to be excluded because they were fussy during the second test session, resulting in too few trials for analysis. All 4 excluded infants provided visual evoked potential (VEP) peaks after stimulus end, preventing us from computing corresponding values for the loom's visual angle and velocity at these times, since these had both become so large after the visual collision had taken place that they were no longer meaningful. Thus, 10 infants ( 6 boys) pro- 
Fig. 1. Experimental set-up with a diagram of stimulus configuration (a) and a timeline of stimuli sequence (b). Each infant was shown a virtual object of a flat circle approaching on a direct collision course. The looming stimulus simulated an object coming from far away approaching for a duration of 2,3 , and $4 \mathrm{~s}$ under three different constant accelerations $(-21.1,-9.4$ and $\left.-5.3 \mathrm{~m} / \mathrm{s}^{2}\right)$. The stimulus approached the infant as the image on the screen grew, and the loom stopped when the image filled the entire screen leaving a blank screen.

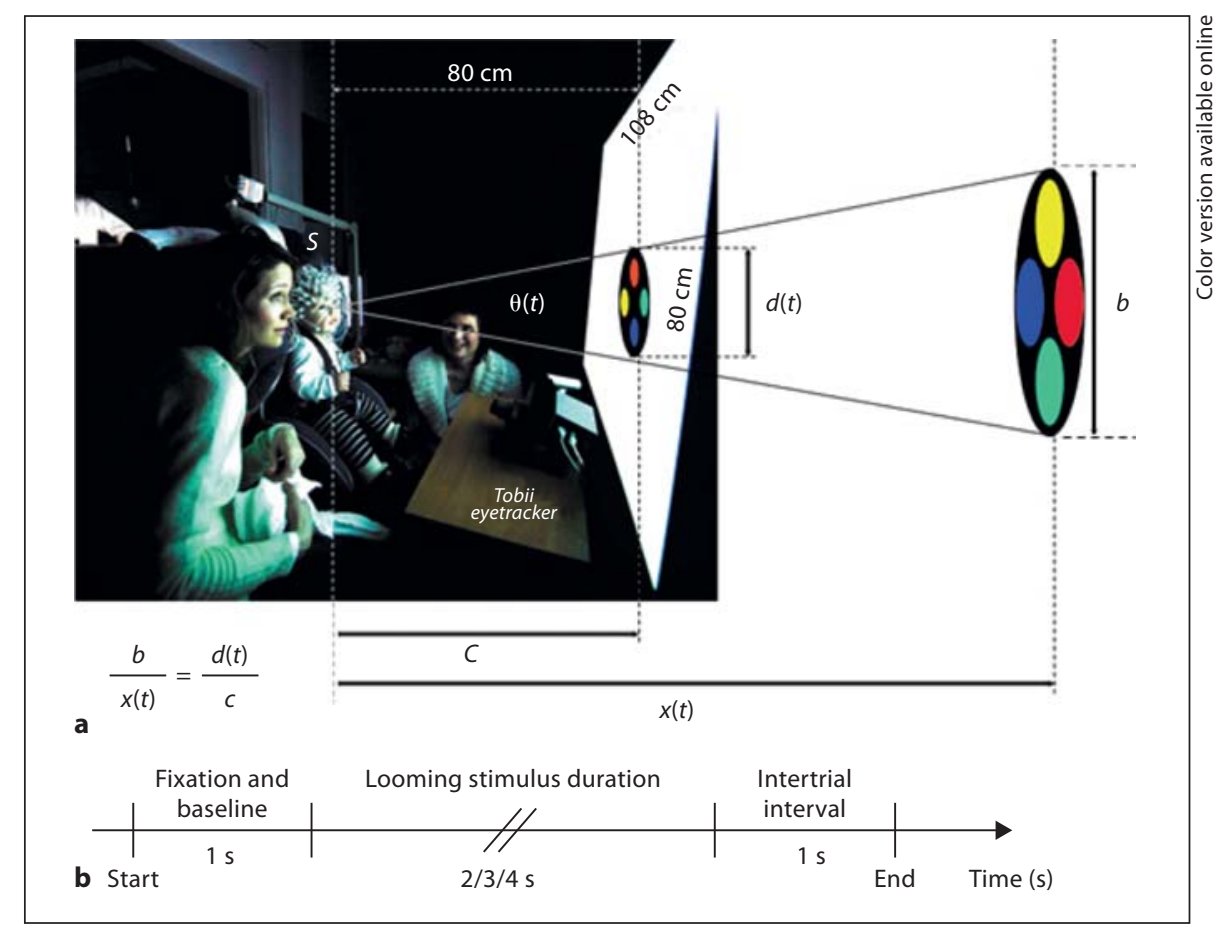

vided data for the final sample and were first tested at an age of $5 / 6$ months, with a mean age of 26 weeks $(S D=1.9)$. The second test session was performed when the infants were about 12/ 13 months, with a mean age of 53 weeks $(S D=2.2)$. At the first session, none of the infants had any crawling experience, but all of them had between 14 and 30 weeks of crawling experience (mean $=21$ weeks, SD =4) the second time they were tested. All infants showed typical development and none had any birth complications as determined by parental report.

EEG recording as a psychophysical procedure causes no physical harm or pain to the participant. The parents gave their informed written consent before the experiment began and they understood that they were free to withdraw at any time. One of the parents was always situated next to the baby during the experimental testing and had the opportunity to interrupt the experiment whenever he/she wanted, whatever the reason. The study has been approved by the Norwegian Regional Ethics Committee and the Norwegian Data Services for the Social Sciences.

\section{Apparatus}

The testing took place in a room with a large projection screen hanging down from the ceiling. The infant was placed with his/ her face $80 \mathrm{~cm}$ away from the screen in a baby car seat. The projection on the screen was $180 \mathrm{~cm}$ wide and $80 \mathrm{~cm}$ high. Corneal reflection (Tobii $\times 50$ ) was used to record the gaze of both eyes of the infant $(50 \mathrm{~Hz})$ and trials in which infants did not look for the entire stimulus duration were excluded from further analyses (fig. 1a; online suppl. movie; for all online suppl. material, see www.karger.com/doi/10.1150/000345154).

EEG activity was recorded with a high-density 126-channel Geodesic Sensor Net 200 [40], run by Electrical Geodesic, Inc. System 200. Arrays of 12-gram Ag sponge sensors were evenly distributed across the head surface. Amplified EEG signals were recorded with Net Station software on a Macintosh computer, using a sampling rate of $500 \mathrm{~Hz}$ with a low-pass filter of $100 \mathrm{~Hz}$ and a $0.1-\mathrm{Hz}$ high-pass filter. All electrode impedances were kept under $50 \mathrm{k} \Omega$, as recommended for the high-input-impedance EGI amplifiers to ensure an optimal signal-to-noise ratio [42, 43]. Triggers about the onset and offset of the looming stimuli were communicated from E-Prime (Psychology Software Tools) onto the EEG recordings of infant brain electrical activity. The data were stored on a hard disc for off-line analyses.

\section{Stimuli}

The stimulus consisted of a flat, black circle with 4 different coloured inner circles of equal size (red, green, blue, yellow) rotating within it. The entire object rotated with a constant angular velocity of 300 degrees per second and was shown on a cream white background. The radius of the inner circles was $1 / 3$ of the radius of the outer circle. The stimuli were programmed to loom towards the infant and coming up to the infant's face so that the infant would get an experience of a visual collision. The virtual object loomed towards the infant with a constant acceleration depending on the condition length. The image of the virtual object appeared on the screen and stayed at its smallest size for $1 \mathrm{~s}$, at a virtual distance of $43.1 \mathrm{~m}$, before it expanded during a looming phase and finally reached its largest size and disappeared, leaving a blank screen for $1 \mathrm{~s}$. The image of the virtual object initially had a visual angle of $5^{\circ}$ (diameter of $6.5 \mathrm{~cm}$ ), and grew to a maximum visual angle of $131^{\circ}$ (diameter of $350 \mathrm{~cm}$ ).

The virtual object approached the infant under three different conditions with a constant acceleration over a period of $2 \mathrm{~s}(-21.1$ $\left.\mathrm{m} / \mathrm{s}^{2}\right), 3 \mathrm{~s}\left(-9.4 \mathrm{~m} / \mathrm{s}^{2}\right)$ and $4 \mathrm{~s}\left(-5.3 \mathrm{~m} / \mathrm{s}^{2}\right)$, respectively. The image of the virtual object had the same size/visual angle at the begin- 


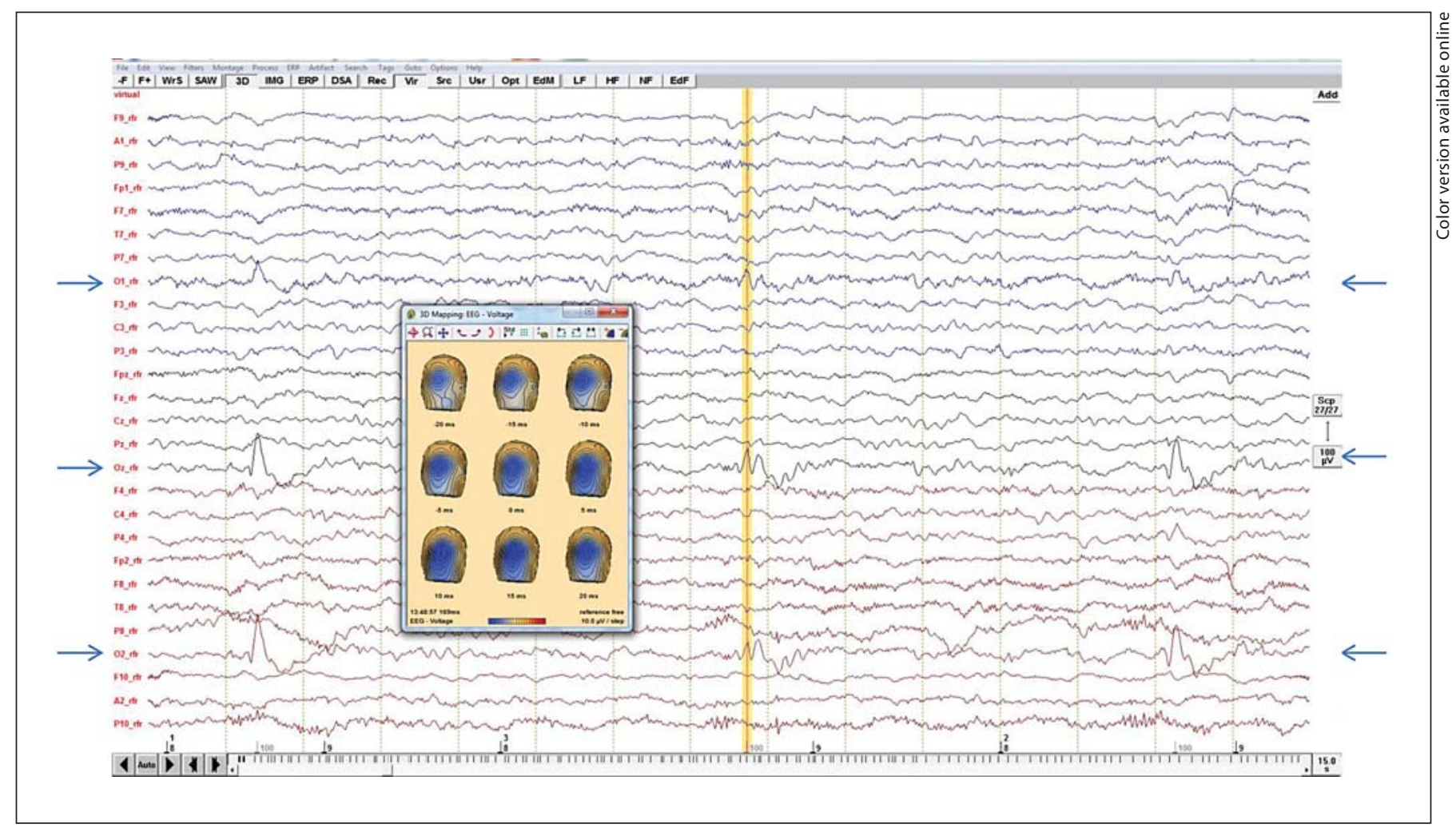

Fig. 2. Raw data from the EEG recordings of a 5-month-old infant displayed using standard 10-20 reference-free sites, showing typical looming-related VEP responses for three random consecutive trials (from left to right: 2,4 , and $3 \mathrm{~s}$ loom, triggers at the bottom alternately indicate stimulus start and stimulus end). VEP peak activity is marked by the vertical line for the 4-second looming trial in the middle. Prominent VEP peaks at sites $\mathrm{O} 1, \mathrm{Oz}$,

ning and the end of the approach, independent of the virtual object's approach time. The virtual object would move over the same virtual distance $(43.1-0.80 \mathrm{~m})$ at a constant acceleration in all three conditions (fig. 1b).

\section{Procedure}

When the baby arrived with its parent(s), they were received by two experimenters. One of them had the responsibility to inform the parent(s) about the experiment, while the other experimenter played with the baby so that they became familiar with each other. When the electrode net was mounted on the infant's head, the infant sat on the parent's lap and was distracted with toys, while the experimenter that was familiarized with the baby put on the electrode net. Immediately after the net was mounted, the infant was placed in a baby car seat facing the screen. One of the parents was always sitting next to the infant to avoid any stress a missing parent may cause, but was instructed to not interfere unnecessarily. In addition, an experimenter was sitting on the other side to rectify and monitor the experiment and to help the baby focus on the screen. The net was plugged into an amplifier and the impedance of the electrodes was checked. Electrodes that had an insufficient and $\mathrm{O} 2$ (indicated with arrows) taking place about $800 \mathrm{~ms}$ before stimulus end can be viewed as direct responses to the looming stimulus in all three trials. The timescale is $1,000 \mathrm{~ms}$ between the thin dotted vertical lines. An EEG voltage build-up and decline over time for the middle trial can be viewed in the inserted 3-dimensional mapping window $(0 \mathrm{~ms}=$ peak VEP response at vertical line).

contact with the scalp were corrected with the use of saline electrolyte or by repositioning them somewhat to improve contact.

The stimuli were generated with E-Prime, and the three different conditions were presented in a random order. Data acquisition was performed in one block, but pauses were made if the infant seemed to have lost interest. The parent and one of the experimenters played with the baby for a couple of minutes before the experiment was continued. The experiment was aborted if no further interest could be obtained or if the infant showed distress.

The experimental room was divided by a window from the control room where the computers for stimulus generation and data acquisition were placed. The experimenter in the control room could observe the infant on a computer screen during the experiment and keep track of whether the infant was paying attention throughout each trial. The experiment ended when the infant lost interest in the stimuli or started fussing.

The looming experiment was performed together with an occlusion experiment and an optic flow experiment, as a means of providing data for other studies. The looming experiment was usually conducted after the optic flow experiment, about 5-7 min into the testing session. 
VEP Responses: Timing and Duration

All EEG data analyses were carried out in the software program Brain Electrical Source Analysis (BESA) 5.1. The procedure was the same for both sessions. First, all EEG recordings were segmented by Net Station and exported to BESA for further analyses. Bad channels and trials contaminated with artefacts from body/ head movement were discarded by visual inspection. None of the participants had more than $10 \%$ of the channels defined as bad. Notch filter was set to $50.0 \mathrm{~Hz}$, and low cut-off filter (high bandpass) was set to $1.6 \mathrm{~Hz}$ to remove slow drift in the data. High cutoff filter (low band-pass) was set to $30 \mathrm{~Hz}$. To be included in the analysis, each infant had to have VEP peaks on 3 trials for each of the 3 looming stimuli, resulting in a minimum of 9 trials for each infant per testing session. The analysis was performed on 424 VEP peaks across all 10 infants, 241 for the first session at 5/6 months and 183 for the second session at 12/13 months.

EEG recordings were used to measure brain electrical changes in the infant brain as groups of neurons fire within the cerebral cortex. This method provides information of the time course and spatial distribution of brain activity due to the visual looming stimulus presented to the infants. A trial-by-trial investigation was performed on each infant's raw EEG recordings. By looking at the reference-free channel distribution, highly prominent VEP peaks were marked at electrode sites $\mathrm{O} 1, \mathrm{Oz}$, and $\mathrm{O} 2$ based on earlier studies investigating VEPs [44]. The marked VEP peaks provided information about the activity at the selected brain regions as a direct measure of amplitude channel activity. This activity could also be visualized by a 3-dimensional mapping of a build-up and a decline of voltage activity in the visual cortex over time. This 3-dimensional mapping and the trial-by-trial visual inspection were used as criteria to determine each VEP peak (fig. 2). At the time each VEP peak occurred, the time remaining to stimulus end which coincided with the loom's time-to-collision was recorded.

Occasionally, several VEP peaks occurred within the same trial so that an additional criterion for VEP peak determination had to be taken into consideration. A more specific localization of brain activity as a response to looming was performed by applying source analysis [45]. A predefined surrogate model for visual areas was used to direct the search of VEPs due to the looming stimuli. This model includes the areas of standard 10-20 sites $\mathrm{O} 1, \mathrm{Oz}$, and $\mathrm{O} 2$ [44]. The BESA algorithm estimated the location and orientation of multiple equivalent dipolar sources by calculating the scalp distribution that would be obtained for a given dipole model (forward solution) and comparing it to the location and orientation in the dipole source. This leads to minimization of the residual variance between the model and the observed spatiotemporal VEP distribution [46]. A 3-shell ellipsoidal head model was created for trials that had less prominent VEP peaks or several peaks [45] and a predefined model of the visual cortex was inserted as dipoles into the head model. In particular two dipoles, VCrL (visual cortex lateral left) and VCrR (visual cortex lateral right), were used to sort out which peaks were a direct response to the looming stimuli $[41,44]$. If the performed source analysis of a certain peak showed a symmetrical synchronized activity at these two dipoles (i.e., source location consistency), this was considered the one that was the response to the looming stimulus.

In addition to recording the timing of the VEP peak with respect to the virtual collision, the duration of every looming-related VEP response was also recorded: VEP duration $=\mathrm{VEP}_{\text {start }}-$ $\mathrm{VEP}_{\text {end }}$ (fig. 3).

Longitudinal Study of Looming in Infants with High-Density EEG

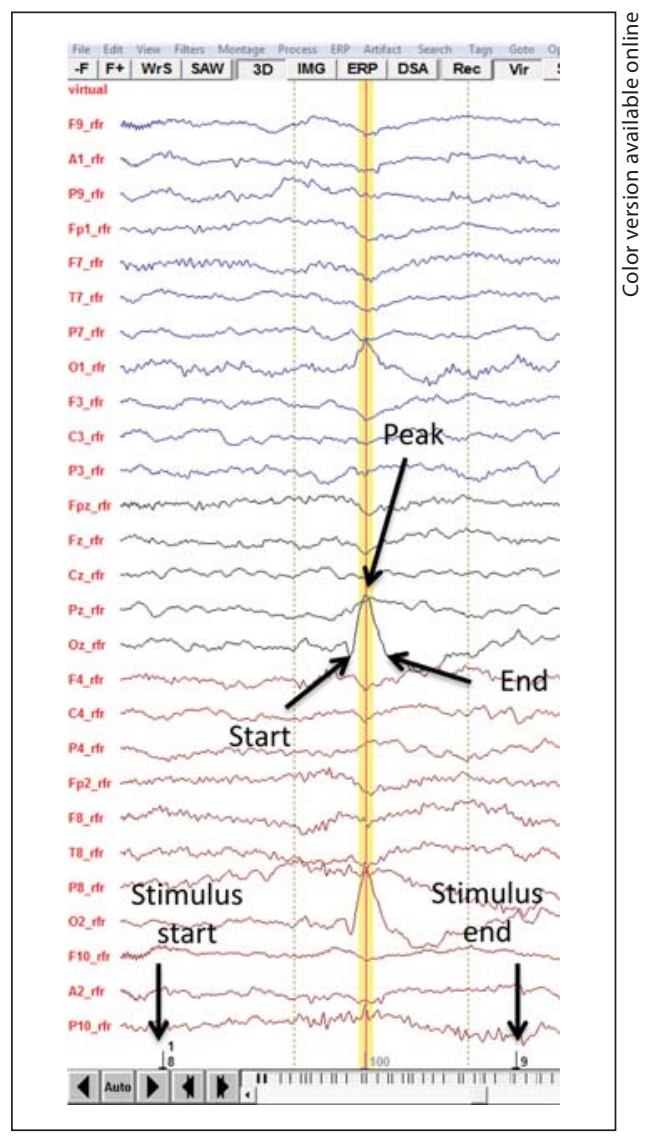

Fig. 3. An example of how VEP duration, defined as $\mathrm{VEP}_{\text {start }}-$ $\mathrm{VEP}_{\text {end }}$, was decided. VEP duration was taken as a measure of processing speed and efficiency.

\section{Timing Strategies}

Infants can use different information variables to estimate the response to the virtually colliding object. Strategies based on the information variables of the colliding object's visual angle, velocity, and time-to-collision were explored in this study. The different information variables would be affected by the stimulus approach velocity and the duration of the approach sequence. By using one information variable to estimate when the virtual collision would take place, there would be expected patterns in the other variables. This can be illustrated by plotting the looming stimulus at an arbitrary point in time during its looming phase (fig. 4). The three graphs show the expected pattern when the brain response is initiated by either one of the three information variables.

A velocity strategy concerns the rate of change of the visual angle, which in this experiment gives the illusion of the approaching loom's forthcoming velocity. By holding this information variable constant (fig. 4a), infants would show a brain response when the loom reaches a certain velocity. As the loom's acceleration decreases the time-to-collision would decrease (fig. 4b), while the visual angle would increase (fig. $4 \mathrm{c}$ ). The visual angle strategy indicates that infants use a certain angle that is subtended by the 


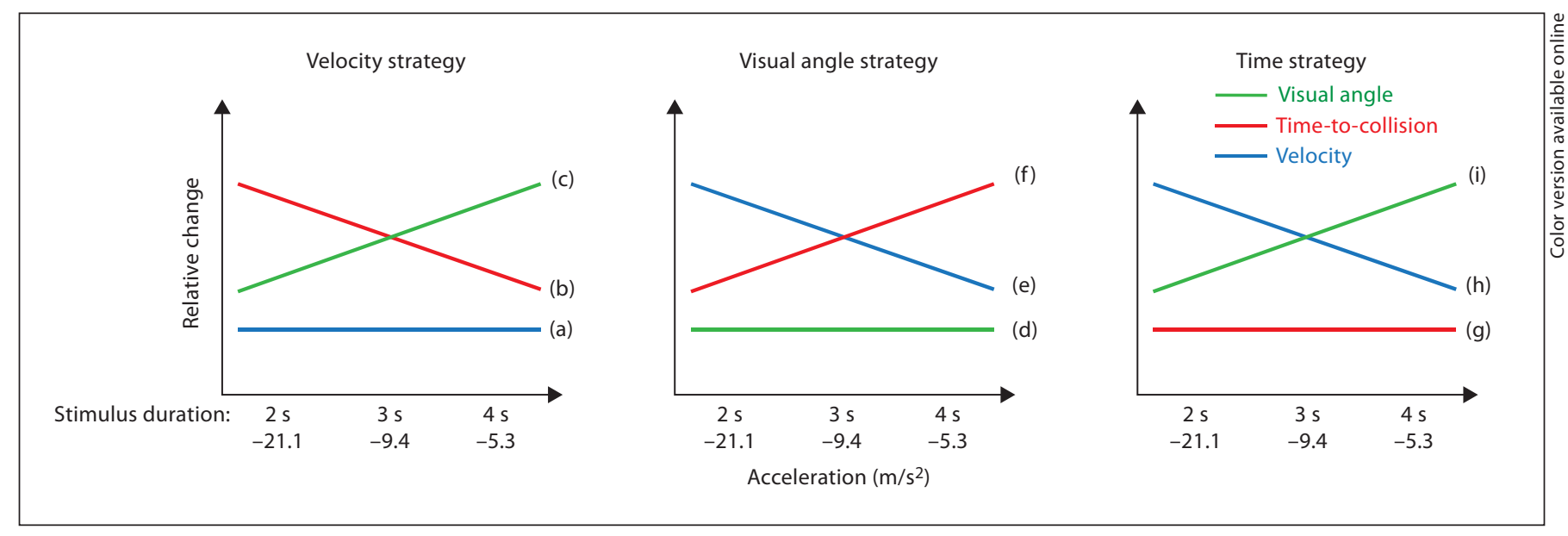

Fig. 4. A theoretical graph of the looming stimulus' velocity, visual angle, and time-to-collision as a function of the duration and acceleration of the looming sequence. As the acceleration of the loom decreases the strategies based on different information variables have different implications for the looming-related brain response. A velocity strategy implies that as the acceleration of the loom decreases, the velocity of the loom is held relatively constant (a), while the loom's time-to-collision decreases (b) and the visual angle increases (c). A visual angle strategy im-

edges of the virtual object at a certain point of observation. If this variable is held constant (fig. 4d) and the acceleration decreases, the loom's velocity would decrease (fig. 4e) while the time-to-collision would increase (fig. 4f).

Either one of these two strategies presented implies that infants respond to a critical value of the loom's visual angle or velocity, respectively. When the object approaches with a constant increase in speed and the duration of the approach varies, information variables based on velocity and visual angle would be influenced by the size and speed of the loom. The loom would reach a certain visual angle or velocity at a different time away from the virtual collision. This makes these optical variables prone to errors when timing a brain response with respect to a collision event so that the response may appear long before or even after the collision would have taken place.

The third strategy investigated was the time strategy, which indicates that the infant shows a brain response to the loom at a specific time before the optical collision. As the loom's acceleration decreases, the time-to-collision is held relatively constant (fig. $4 \mathrm{~g}$ ), while the velocity would decrease (fig. $4 \mathrm{~h}$ ) and the visual angle would increase (fig. 4i). This information variable is not dependent on knowledge of object size or speed, so the usage of a time strategy would always leave the same amount of time for the brain response. The time strategy is considered to be a more efficient strategy that is less prone to errors compared to the two other strategies investigated [35-37].

At the time every selected VEP peak occurred, corresponding values for the loom's visual angle, velocity, and time-to-collision were computed. In order to explore which timing strategy infants used to time their brain responses to the virtual collision, values plies that as the acceleration of the loom decreases, the visual angle of the loom is held relatively constant (d), while the loom's velocity decreases (e) and time-to-collision increases (f). A time strategy implies that as the acceleration of the loom decreases, the loom's time away from the virtual collision is kept relatively constant (g), while the loom's velocity decreases (h) and the visual angle increases (i). For simplicity, the lines are drawn as straight lines. For colours, see online version.

for all variables were averaged for each infant across the three looming conditions. It was assumed that the variable with the lowest index of dispersion (ID = standard deviation divided by the mean), also referred to as the coefficient of variation, was the one controlled. When adhering strictly to one strategy, there would be consequences for the other strategies, as the strategies are mutually exclusive. By holding one variable constant, the values of the other two variables would vary [36].

\section{Results}

\section{Averaged Waveforms}

So as to give an impression of the kind of data that were subjected to the trial-by-trial analysis below, averaged waveforms of two typical infants' VEP responses to the looms for the two testing sessions at 5/6 months and 12/13 months are presented in figure 5 . It can be seen that averaged looming-related responses were most pronounced in occipital channels $\mathrm{O} 1, \mathrm{Oz}$, and $\mathrm{O} 2$, and that the activity propagated to parietal channels $\mathrm{P} 3$ and $\mathrm{Pz}$ particularly at the second session.

\section{VEP Responses: Timing and Duration}

Infants were very impressed with the looming stimuli and looming-related VEPs were found in the EEG read- 
ings from all of the tested infants where the stimulus was approaching the infant on a direct collision course. On average, each infant contributed $24(\mathrm{SD}=4.0)$ VEPs in the first session and $18(\mathrm{SD}=7.1)$ in the second session. Looming-related peak VEP responses in the occipital areas $\mathrm{O} 1, \mathrm{Oz}$, and $\mathrm{O} 2$ were indexed as the times before the loom would have made contact with the infant, i.e. the time-to-collision. A 2 (age: 5/6 vs. 12/13 months) $\times 3$ (loom: 2, 3, 4 s) repeated-measures ANOVA was performed on averaged peak VEP responses. This revealed a main effect of age $[F(1,9)=6.072, p<0.05]$, indicating that averaged peak VEP responses occurred significantly closer to the virtual collision with the looming stimulus for infants at the age of $12 / 13$ months $(-0.673 \mathrm{~s}, \mathrm{SD}=$ $0.275)$ than for infants at the age of $5 / 6$ months $(-0.914 \mathrm{~s}$, $\mathrm{SD}=0.148)$ (fig. 6).

Another 2 (age: 5/6 vs. 12/13 months) $\times 3$ (loom: 2, 3, $4 \mathrm{~s})$ repeated-measures ANOVA was performed on averaged VEP duration $\left(\mathrm{VEP}_{\text {start }}-\mathrm{VEP}_{\text {end }}\right)$. A main effect of age was found on averaged VEP duration $[\mathrm{F}(1,9)=6.913$, $\mathrm{p}<0.05$ ], indicating that averaged VEP duration at $12 / 13$ months $(0.152 \mathrm{~s}, \mathrm{SD}=0.036)$ was significantly shorter than averaged VEP duration at 5/6 months $(0.187 \mathrm{~s}, \mathrm{SD}=$ 0.042) (fig. 7).

Findings from the two ANOVAs indicate that the averaged peak VEP response appears earlier in the looming sequence and has a longer VEP duration when infants are $5 / 6$ months old as opposed to when they are 12/13 months old. At 12/13 months, this response is closer to the loom's virtual collision and of a shorter duration. There were no significant main effects of loom, nor any interaction effects in either of the two ANOVAs performed.

\section{Timing Strategies}

At the time each VEP peak occurred, the loom's visual angle, velocity, and time away from collision were computed and an ID analysis was performed across looms on these values for each infant at both sessions (table 1). The ID indicates how much a score deviates from its central tendency and is found by dividing the standard deviation by the mean. It is a dimensionless number, allowing the direct comparison between the three timing strategies. When exploring which information variable the infants used in order to estimate the loom's time of arrival, it was assumed that the strategy the infants held constant across the different approach conditions was the one they employed. The information variable with the smallest ID was taken to be the variable controlled by the infant $[37,47,48]$. Based on their ID, there were 4 infants that used a velocity strategy and 6 infants that used a time

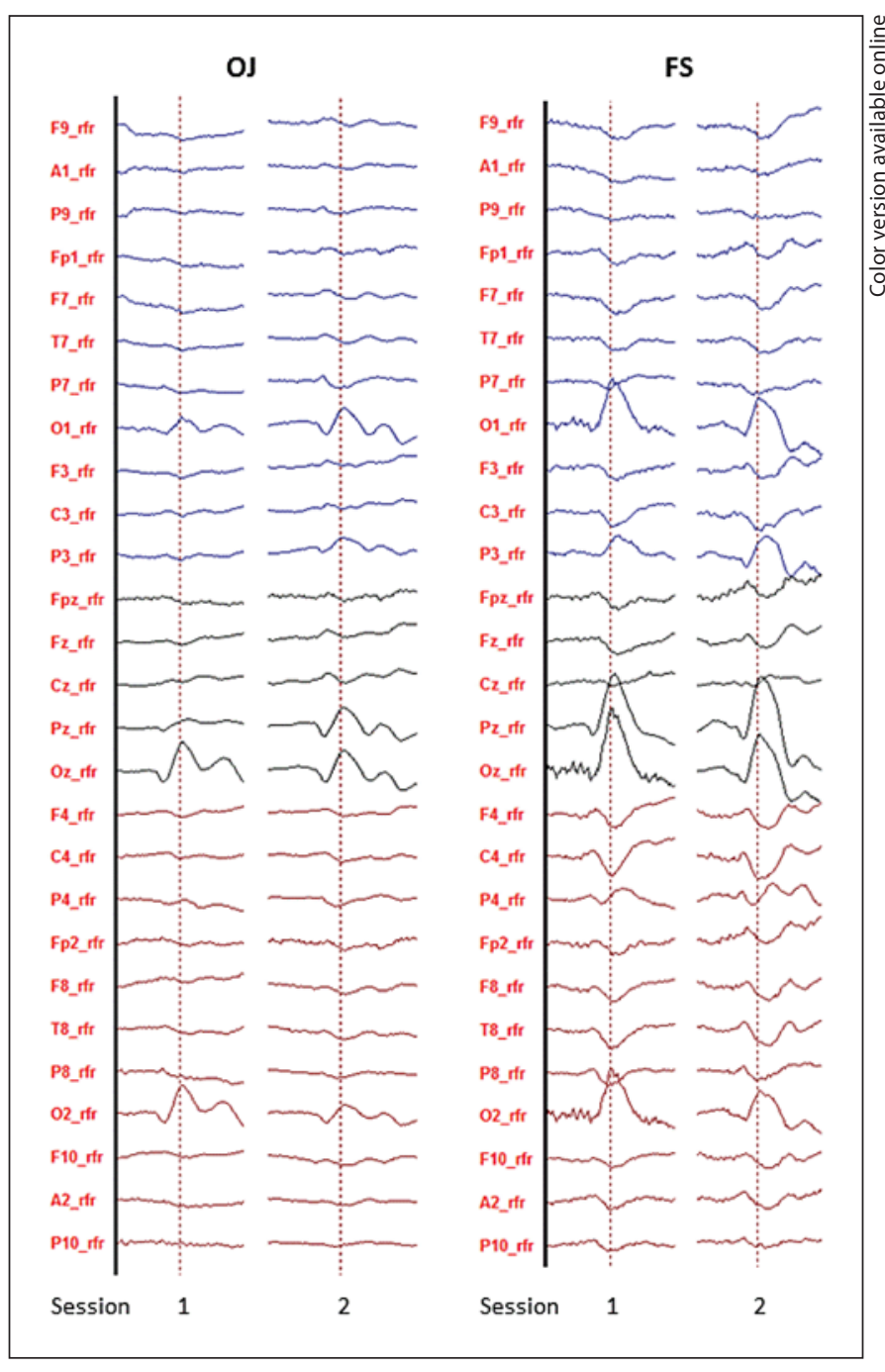

Fig. 5. Averaged waveforms across the 3 loom speeds for 2 typical infants at 5/6 months and at $12 / 13$ months using a virtual montage where the standard 27 interpolated channels are displayed against a reference-free average reference. Note the prominent loomingrelated VEP responses in occipital channels $\mathrm{O} 1, \mathrm{Oz}$, and $\mathrm{O} 2$ and, in addition, in parietal channels $\mathrm{P} 3$ and $\mathrm{Pz}$ especially at the second session. Both infants contributed with between 25 and 30 trials per session. The timescale on the $\mathrm{x}$-axis is $500 \mathrm{~ms}$.

strategy at the age of 5/6 months. The ID calculations of the second session showed that 9 out of 10 infants used a time strategy at the age of $12 / 13$ months. These findings indicate a strategy shift from a less efficient strategy to a more efficient strategy in 4 of the infants. One of the infants (infant U.L.; table 1) showed an opposite pattern of strategy shift and went from a time strategy to a velocity strategy. 


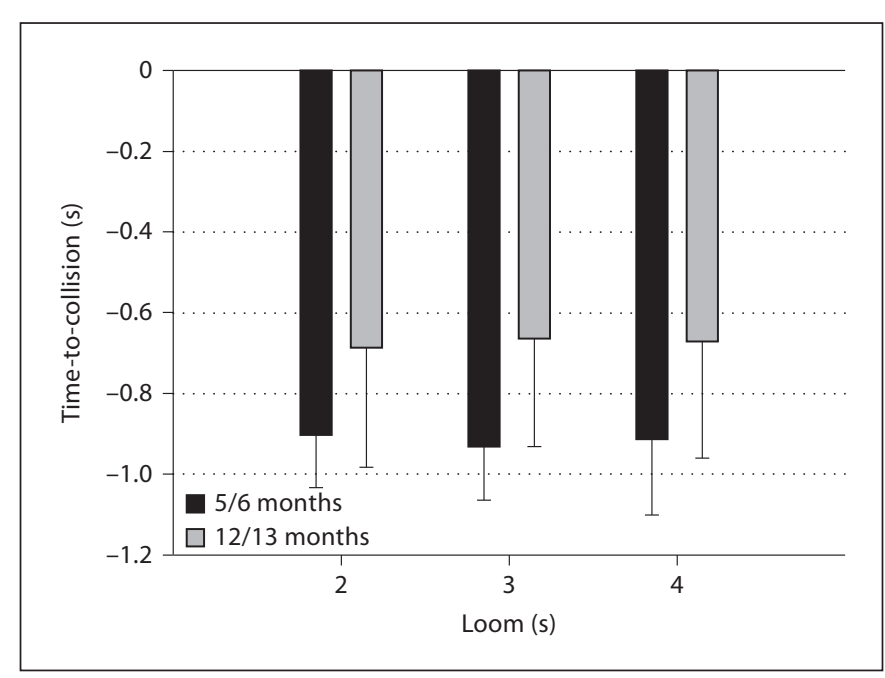

Fig. 6. Timing of averaged looming-related peak VEP responses (including SD bars) for the 3 looms at the age of 5/6 months and at the age of $12 / 13$ months. With age, infants time their VEP peaks significantly closer to the loom's virtual collision compared to when they were younger.

Table 1. Index of dispersion

\begin{tabular}{|c|c|c|c|c|c|c|}
\hline \multirow{3}{*}{$\begin{array}{l}\text { Infant } \\
\text { O.J. }\end{array}$} & \multicolumn{3}{|c|}{$5 / 6$ months old } & \multicolumn{3}{|c|}{$12 / 13$ months old } \\
\hline & \multirow{2}{*}{$\begin{array}{c}\begin{array}{c}\text { visual } \\
\text { angle }\end{array} \\
0.24\end{array}$} & \multicolumn{2}{|c|}{ velocity time } & \multirow{2}{*}{$\begin{array}{c}\begin{array}{c}\text { visual } \\
\text { angle }\end{array} \\
0.23\end{array}$} & \multicolumn{2}{|c|}{ velocity time } \\
\hline & & 0.18 & 0.13 & & 0.28 & 0.08 \\
\hline T.M. & 0.34 & 0.17 & 0.21 & 0.23 & 0.17 & 0.06 \\
\hline F.S. & 0.46 & 0.17 & 0.24 & 0.22 & 0.13 & 0.01 \\
\hline I.O. & 0.31 & 0.22 & 0.17 & 0.27 & 0.25 & 0.24 \\
\hline L.P. & 0.27 & 0.21 & 0.24 & 0.28 & 0.26 & 0.14 \\
\hline R.I. & 0.21 & 0.11 & 0.06 & 0.18 & 0.15 & 0.08 \\
\hline V.L. & 0.32 & 0.16 & 0.19 & 0.24 & 0.25 & 0.04 \\
\hline L.T. & 0.21 & 0.11 & 0.05 & 0.24 & 0.19 & 0.11 \\
\hline U.L. & 0.20 & 0.16 & 0.12 & 0.42 & 0.13 & 0.25 \\
\hline R.M. & 0.23 & 0.12 & 0.07 & 0.24 & 0.17 & 0.23 \\
\hline
\end{tabular}

For each infant at both ages tested, an ID analysis was carried out to allow a direct comparison of the optical variables of visual angle, velocity, and time-to-collision so as to assess which of these three variables the looming-related VEP responses were geared to. A small ID indicates little variation across the different approach conditions. The lowest ID is highlighted in italics for each infant and is, in turn, interpreted as the variable being controlled by the infant. Please note that by the age of $12 / 13$ months, all infants except 1 appear to be gearing their looming-related VEP responses to the loom's time-to-collision.

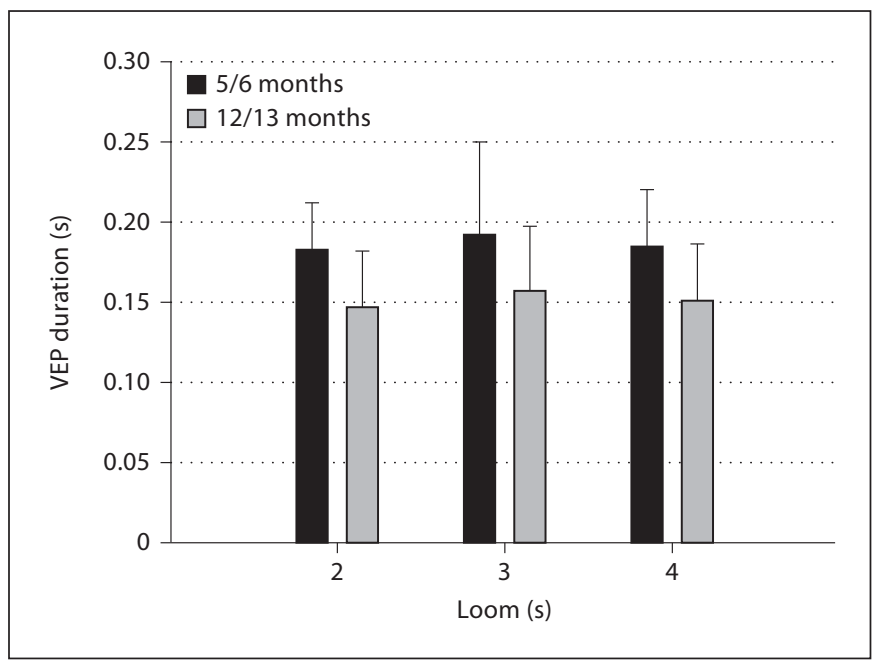

Fig. 7. Averaged VEP duration, $\mathrm{VEP}_{\text {start }}-\mathrm{VEP}_{\text {end }}$ (including SD bars) for the 3 looms at the age of $5 / 6$ months and at the age of $12 / 13$ months. With age, infants show significantly shorter VEP durations compared to when they were younger.

\section{VEP Amplitudes}

Averages of looming-related peak VEP activation were computed for each infant for both testing sessions. The individual participant averages were referenced to an artificial reference calculated from the average potentials over the scalp and interpolated to 81 standard electrode positions. These interpolated files consisting of individual participant averages were combined into a grand average for infants aged 5/6 months and compared with the grand average for the same infants aged $12 / 13$ months. A 2 (age: 5/6 vs. $12 / 13$ months) $\times 3$ (area: $\mathrm{O}, \mathrm{PO}, \mathrm{P}$ ) repeated-measures ANOVA was performed on averaged peak VEP activation. The investigated electrode areas were selected based on anatomical positioning of the brain areas involved in visual processing $[10-13,15-17]$ and on the grounds of visual inspection of the two grand averages. The results showed an interaction effect between age and cortical area $[\mathrm{F}(2,18)=5.982, \mathrm{p}<0.01]$, indicating that the activity in our investigated occipital and parietal areas differed between the measurements performed at 5/6 months and 12/13 months. The amplitude activity in the $\mathrm{O}$ area decreased from $5 / 6$ to $12 / 13$ months, while the activity in the $\mathrm{P}$ area increased from $5 / 6$ to $12 / 13$ months. The amplitude activity in the PO area in between stayed relatively the same at the two ages (fig. 8). 


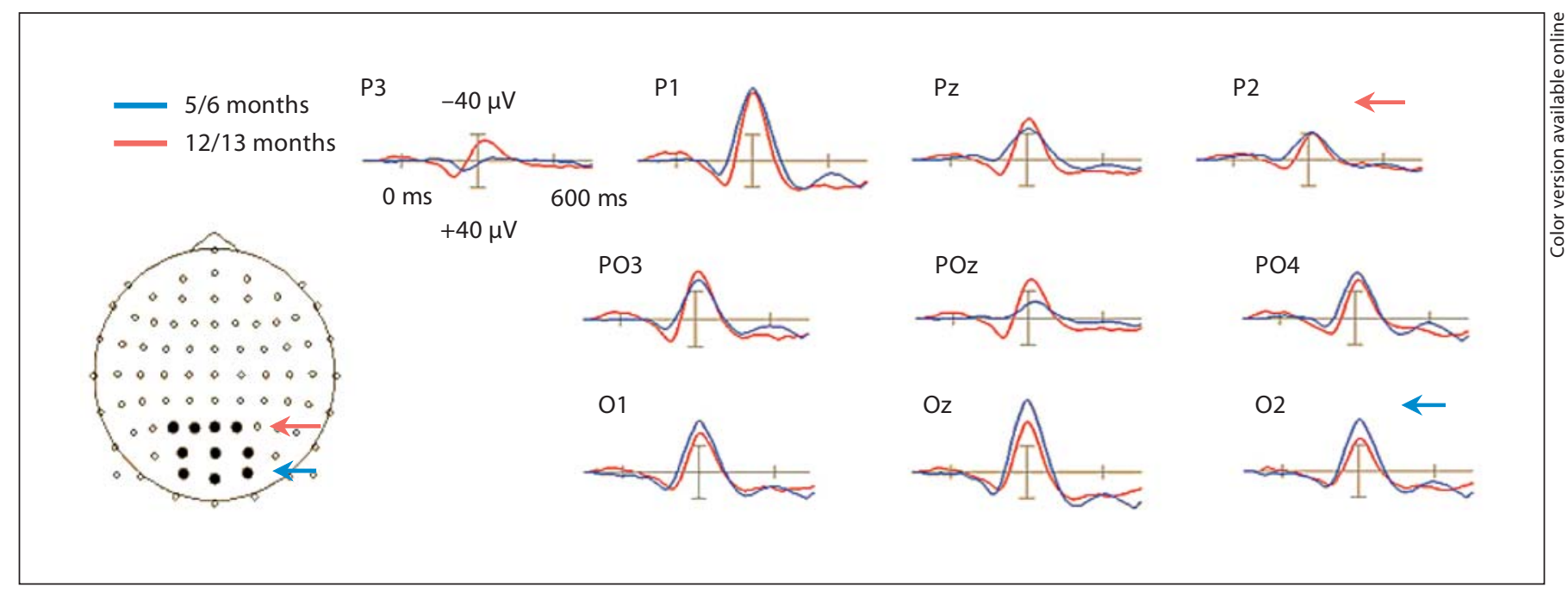

Fig. 8. Grand average looming-related VEP activity for infants aged 5/6 months and 12/13 months. Scalp localization of the electrode sites from bottom to top and from left to right for the two ages $(\mathrm{O} 1, \mathrm{Oz}, \mathrm{O} 2, \mathrm{PO} 3, \mathrm{POz}, \mathrm{PO} 4, \mathrm{P} 3, \mathrm{P} 1, \mathrm{Pz}, \mathrm{P} 2)$ is indicated with the filled circles on the head drawing (nose up). Infants at the age of 5/6 months show a larger negative amplitude activity in the $\mathrm{O}$ areas (bottom arrow) compared to when they were 12/13 months. At the age of 12/13 months, on the other hand, infants show a larger negative activity in the $\mathrm{P}$ areas (top arrow) compared to when they were 5/6 months. For colours, see online version.

\section{Discussion}

In order to respond adequately to a looming visual object approaching on a direct collision course, infants have to perceive the collision before it happens, which requires prospective control with regards to the forthcoming collision. This kind of processing would demand highly efficient systems that are able to integrate visual information with action in order to produce an appropriate response [1-4]. Such prospective control is fundamental when timing interceptive actions. The present longitudinal study investigated the development of motion processing of a looming object in infants aged 5/6 and 12/13 months. While infants observed a stimulus looming towards them on a direct collision course under three different accelerations, their looming-related brain responses were measured through EEG recordings.

\section{VEP Responses: Timing and Duration}

When investigating the timing of the looming-related averaged peak VEP responses with respect to the virtual collision, infants at the age of 5/6 months showed an average peak VEP at $0.914 \mathrm{~s}$ before the virtual collision, compared to $0.673 \mathrm{~s}$ at the age of $12 / 13$ months. Infants at the age of 5/6 months thus showed a peak VEP on average $0.241 \mathrm{~s}$ earlier in the looming sequence compared to at $12 / 13$ months. Irrespective of the loom's three different approach conditions, at 5/6 months the looming-related brain responses appear relatively early in the looming phase, when the looming object is still far from the infant's face. At 12/13 months, however, infants' VEP peaks appear much closer to the virtual collision. The peak VEP responses found early in the looming sequence at the age of 5/6 months could be a result of not yet fully processed visual information. Neural pathways at the age of 5/6 months are generally less specialized compared to neural pathways developed in the infant brain at the age of 12/13 months. As a result of immature cortical pathways within the visual processing route, cortical regions may respond to a wide variety of visual stimuli. This implies that several pathways become partially activated; consequently, young infants' response actions are often based on outputs conducted from the first available brain pathway. This can occur when a task situation is complex or demanding and responses become deteriorated or incorrect [34]. Through development, the same region gets more specifically tuned to certain stimuli or situations so that neural pathways only become engaged by a subset of these stimuli, which would lead to a more accurate response $[34,49]$. This can be one of the explanations of the findings in our data showing more accurately timed VEP peaks with respect to the virtual collision at $12 / 13$ months compared to at 5/6 months. 
Infants in our study showed looming-related VEPs not only earlier in the looming sequence, but also of a slightly yet significantly longer mean duration of $35 \mathrm{~ms}$ at 5/6 months compared to at $12 / 13$ months. A VEP response closer to the virtual collision and of a shorter duration could indicate an increase in speed and efficiency of the processing of visual information [31-34, $41,50]$. During the first year of life, the infant brain more than doubles in weight simultaneously as it undergoes rapid changes $[34,50]$, suggesting that infants at the age of 5/6 months generally have a less developed brain than at the age of 12/13 months. Looming-related VEPs timed closer to the loom's virtual collision and of a shorter duration could indicate a developmental trend in our findings.

Several underlying mechanisms connected to infants' functional brain development can be used to explain these findings. An important physiological change that is associated with more efficient cortical processing is an increase in white matter due to myelination of axons [30, $31,51]$. Through experience of certain stimuli or situations, the brain becomes tuned and myelination of axons between neurons appears. The conductivity velocity between pathways increases mainly due to myelination, which indicates that the processing speed also increases [50]. Infants in our data showed a decrease in VEP duration from 5/6 months to $12 / 13$ months. This decrease could indicate that as infants get older they require less time to process looming stimuli which could be related to improved synchronization of responses across occipital and parietal regions.

Infants' functional brain development is probably best explained by looking at multiple coactive and differential specialized neural pathways [34]. When pathways in the brain get specialized to a certain type of stimulus, a smaller brain area is activated and the processing speed increases. This specialization may occur in relation to several physiological changes in the brain. Maturational development of the infant brain could be an outcome of synaptic maturation through neural selection and competition between neural pathways. As connections between pathways are more easily stabilized between nearby pathways than pathways separated by a longer distance, close-by pathways are more likely to be favoured over other pathways. The distance between pathways together with a correspondence between pathways' structures and functions concerns a strengthening of some connections and pruning of other connections. Strengthening and pruning of neural pathways could be one of the developmental changes that affect processing speed and efficiency as a smaller brain area becomes activated as neural pathways become more specialized to a certain stimulus $[34,49]$.

\section{Timing Strategies}

The ID analysis conducted on infants' use of timing strategy when estimating their VEP response with respect to the looming stimulus showed that 6 of the infants used a time strategy and 4 used a velocity strategy at the age of 5/6 months. This implies that 6 of the infants already used a strategy that was supposedly based on a more efficient information variable compared to the other strategies investigated. Only 4 of the infants showed a strategy shift from a less efficient strategy to a more efficient strategy. These infants switched from a velocity strategy to a time strategy between the ages of 5/6 and $12 / 13$ months. Interestingly, the only infant who at the age of $12 / 13$ months appeared to be using a velocity strategy had started rolling over relatively late at 28 weeks and had between 1 month to up to almost 4 months less crawling experience than the other infants.

Using a strategy based on time, infants' brain electrical response becomes less prone to errors associated with the looming object's acceleration. By timing the response a certain time away from the loom's virtual collision, infants would always have the same amount of time to make a response independent of the loom's acceleration $[35,36]$. However, infants' brain responses to looming would be influenced by when in the looming sequence the response is estimated $[47,52]$. If the estimate of the time-to-collision appears early in the looming sequence, there is more room to make impoverished or incorrect judgments. By delaying the response, other relevant information from the loom's accelerative approach could be taken into account to make a more accurate response [34, $36,52,53]$. For example, an estimate made according to the loom's acceleration early in the sequence would probably lead to a less accurate interpretation of the loom's acceleration and thus change in velocity as it approaches the baby. The possible loss of important information about the loom's acceleration when responding too early in the looming sequence can cause an incorrectly estimated defensive response with respect to the loom's virtual collision.

At $12 / 13$ months, infants showed VEP peaks on average $0.241 \mathrm{~s}$ closer to the virtual collision than at 5/6 months. As argued above, this would lead to more inaccurate responses at 5/6 months which could be explained by developmental factors such as unspecialized and less mature pathways in the infant brain at this age [34]. By 
just looking at the ID analysis, it is difficult to point out differences between infants in the two strategy groups. The observed shift in strategy between infants using a velocity strategy at 5/6 months and a time strategy at $12 / 13$ months could nevertheless imply maturation and specialization of brain pathways. The shift from a less to a more efficient strategy concurrent with a more efficient employment of the used timing strategy, as evidenced by the VEPs occurring closer to the virtual collision, provides further evidence for the developmental trend found in our data. An increase in accuracy and efficiency of an output response indicates further processing of visual information and specialization of brain pathways [34, 50].

\section{VEP Amplitudes in the $O, P O$, and $P$ Areas}

Infants' awareness of a looming object on collision course is well established through behavioural studies of the blinking response $[19,20,35-37]$, but less is known about infants' specialization in visual pathways of looming motion. The dorsal stream in the adult cortex (V1, V2, $\mathrm{MT}+$ ) has neurons that are selectively activated by motion stimuli [15-17], and studies of the human visual system have provided growing evidence that the visual system is specialized to detect and respond to looming motion [1113]. The results from our study concerning averaged peak VEP activity at the cortical areas $\mathrm{O}, \mathrm{PO}$, and $\mathrm{P}$ showed that the activity at these sites varied according to the infant's age. For infants aged 5/6 months, the activation in the $\mathrm{O}$ area showed larger negative amplitudes than at the age of 12/13 months. An opposite pattern was shown in the peak amplitudes in the $\mathrm{P}$ area at 12/13 months, implying that infants had larger negative amplitudes in the $\mathrm{P}$ area at $12 / 13$ months than at 5/6 months. As mentioned above, larger brain areas show a stimulus-specific effect when the infant lacks experience with a stimulus or situation. An increase in experience to certain stimuli or situations would lead to specialization of the pathways involved in the processing, whereas others become inhibited. This change of localization can be viewed as a direct consequence of specialization as fewer pathways become activated by a given stimulus $[34,49,50]$. This explanation may correspond with our findings from the averaged peak VEP activation analysis together with findings from adult data which show that the processing of motion stimuli is propagated along the dorsal stream towards the $\mathrm{MT}+$ area of the brain [11-13]. The decrease in activity in the $\mathrm{O}$ area and increase in the $\mathrm{P}$ area with age may imply that activation in occipital areas propagates forward and starts to influence more advanced areas in parietal and temporal leads [31]. The cortical areas composing the dorsal stream appear to be arranged due to a progression of areas in which a more complex or specialized visual processing develops [54].

Findings of the propagated amplitude activation between the different areas of the brain as well as findings of an increased processing speed could indicate that the visual pathway has become more specialized with age from $5 / 6$ to $12 / 13$ months. This assumption is also supported by other findings where an increase in processing speed of a visual stimulus in infants between 1 and 4 months was observed, suggesting that there is a strong correlation between microstructural maturation of optic radiations and the functional development of visual perception during early infancy [33]. Myelination of the visual pathway was considered to play a major role in the increase in processing speed found in their study.

A direct comparison of electrode site activity is very likely to have been influenced by activation from neural sites other than those accounted for in our investigation. Because EEG measures the summation of neural activity that has propagated to the scalp, it may not adequately separate sources that temporally overlap. Our finding of the propagated activity could therefore be a result of the temporal summation of changes in other components [31]. It could also occur because of additional processes emerging that are not yet identified or taken into consideration.

Another factor that could have influenced our findings of differences in amplitude activation between the two age groups could be the result of an increase in skull thickness. Infant skull thickness increases most rapidly within the first year of life $[30,55]$, resulting in a thicker skull at 12/13 months than at 5/6 months. Because of the skull's poor conductivity, it is possible to argue that the decrease in activity at the $\mathrm{O}$ area may have been caused by an increase in skull thickness from the age of 5/6 months to the age of 12/13 months, instead of being a result of changed localization for more specialized and advanced processing of the visual information. On the other hand, this argument would become inadequate when applied to the findings from the increased activity in the $P$ area with age. Despite the fact that the infants' skulls became significantly thicker as they aged from 5/6 months to 12/13 months, we still found an increase in averaged VEP activity in the P area as they became older. This supports the argument that infants' processing of the looming stimuli propagates from occipital towards more specialized and advanced parietal neural pathways. 


\section{General Discussion}

In this study, infant brain electrical activity was investigated before any of the infants had started to crawl and then again after 5 months of crawling experience had been achieved. In a perspective where structural brain development and perceptual, cognitive, and behavioural changes are integrated in order to fully understand human functional development, brain and behavioural development become reciprocally dependent [34]. One of the differences between the infants at the two different ages was the ability to locomote. When aged 12/13 months, all of the infants had ample crawling experience, whereas none of them could crawl when aged 5/6 months. Self-produced motor activity has been linked to psychological development by a number of theories $[24,28,56]$. These studies argue that motor experience can influence whether available information about a stimulus will be noticed and used by the perceiver. Experience related to self-produced locomotion may permit infants to convert effective information variables available in the optical flow field into information useful for prospective control [5]. Based on the view of an interdependent relationship between brain and behavioural development it is possible to argue that as infants get more experience with selfproduced locomotion, the ability to more accurately process information about an impending virtual collision will improve [41]. Changes in locomotor development could thus be one of the underlying causes of the developmental trend observed in our data.

In conclusion, younger infants not yet capable of crawling showed different brain responses to a looming stimulus approaching on a direct collision course, compared with when they had several weeks of crawling ex- perience. First of all, the present study identified looming-related brain electrical responses in the raw data from the EEG recordings in infants. The study further showed clear differences in VEP responses as infants got older, shown by a decrease in processing time and VEP peaks closer to the loom's time-to-collision. The measured peak VEP activation also propagated towards higher information processing areas in the visual pathway. Infants further showed a shift from a less efficient to a more efficient timing strategy involving the loom's time-to-collision between the ages of 5/6 months to 12/13 months. The findings indicate a developmental trend in infants' prediction of an object's time-to-collision. The observed differences can reflect both a more mature anatomy as well as a higher degree of specialization of brain pathways in the infant brain at around 1 year of age. It is also possible to argue that changes in behavioural development together with changes in the developing brain could be the underlying causes of this trend. Our study of the development of prospectively judging when a looming object will collide by using EEG recordings may contribute to a deeper understanding of the neural functions underlying the development of visual motion perception.

\section{Acknowledgments}

The research presented in this paper was part of a Master's thesis by Monica Svantesson. The authors acknowledge the assistance of Jan Frederik Léger and Sanne Houweling in programming the visual stimuli, and are grateful to Magnus Holth for his help with testing. We would like to thank all infants and their parents who took part in the work and without whom this study would not have been possible.

\section{References}

1 Wilkie R, Wann JP: Controlling steering and judging heading: retinal flow, visual direction, and extraretinal information. J Exp Psychol Hum Percept Perform 2003;29:363378.

2 Von Hofsten C: An action perspective on motor development. Trends Cogn Sci 2004; $8: 217-231$.

-3 Von Hofsten C: Action in development. Dev Sci 2007; 10:54-60.

4 Lee DN: Guiding movement by coupling taus. Ecol Psychol 1998;10:221-225.

5 Gibson JJ: The Ecological Approach to Visual Perception. Boston, Houghton Mifflin Company, 1979.
6 Von Hofsten C, Rosander K: Development of smooth pursuit tracking in young infants. Vision Res 1997;37:1799-1810.

7 Shea SL, Aslin RN: Oculomotor responses to step-ramp targets by young human infants. Vision Res 1990;30:1077-1092.

8 Zeki SM: Functional organization of a visual area in the posterior bank of the superior temporal sulcus of the rhesus monkey. J Physiol 1974;236:549-573.

$\checkmark 9$ Zeki SM: Thirty years of a very special area: area V5. J Physiol 2004;551:1-2.

10 Dougherty RF, Koch VM, Brewer AA, Fischer B, Modersitzki J, Wandell BA: Visual field representations and locations of visual areas $\mathrm{V} 1 / 2 / 3$ in human visual cortex. J Vision 2003;3:586-598.
Holliday IE, Meese TS: Neuromagnetic evoked responses to complex motions are greatest for expansion. Int J Psychophysiol 2005;55:145-157.

12 Morrone MC, Tosetti M, Montanaro D, Fiorentini A, Cioni G, Burr DC: A cortical area that responds specifically to optic flow, revealed by fMRI. Nat Neurosci 2000;3:13221328.

13 Shirai N, Yamaguchi MK: Asymmetry in the perception of motion-in-depth. Vision Res 2004;44:1003-1011.

14 Proverbio AM, Del Zotto M, Zani A: Interindividual differences in the polarity of early visual responses and attention effects. Neurosci Lett 2007;419:131-136. 
15 Braddick O, Birtles D, Wattam-Bell J, Atkinson J: Motion- and orientation specific cortical responses in infancy. Vision Res 2005;45: 3169-3179.

-16 Cheng K., Fujita H, Hanno I, Miura S, Tanaka K: Human cortical regions activated by wide-field visual motion: an $\mathrm{H}_{2}{ }^{15} \mathrm{O}$ PET study. J Neurophysiol 1995;74:413-427.

-17 Rosander N, Nyström P, Gredebäck G, Von Hofsten C: Cortical processing of visual motion in young infants. Vision Res 2007;47: 1614-1623.

18 Bower TGR, Broughton JM, Moore MK: Infant responses to approaching objects: an indicator of response of distal variables. Percept Psychophys 1970;9:193-196.

19 Schiff W, Caviness JA, Gibson JJ: Persistent fear responses in rhesus monkeys to the optical stimulus of 'looming'. Science 1962;136: 982-983.

20 Yonas A, Pettersen L, Lockman JJ: Young infants' sensibility to optical information of collision. Can J Psychol 1979;33:268-279.

21 Hatsopoulos N, Gabbiani F, Laurent G: Elementary computation of object approach by wide-field visual neuron. Science 1995;270: 1000-1003.

22 Martinoya C, Delius JD: Perception of rotating spiral patterns by pigeons. Biol Cybern 1990;63:127-134.

-23 Sun H, Frost BJ: Computation of different optical variables of looming objects in pigeon nucleus rotundus neurons. Nat Neurosci 1998;1:296-202.

-24 Anderson DI, Campos JJ, Anderson DE, Thomas TD, Witherington DC, Uchiyama I, Barbu-Roth MA: The flip side of perceptionaction coupling: locomotor experience and the ontogeny of visual-perceptual coupling. Hum Mov Sci 2001;20:461-487.

$\checkmark 25$ Bell MA, Fox NA: Crawling experience is related to changes in cortical organization during infancy: evidence from EEG coherence. Dev Psychobiol 1996;29:551-561.

26 Bell MA, Fox NA: Individual differences in object permanence performance at 8 months: locomotor experience and brain electrical activity. Dev Psychobiol 1998;31:287-297.

27 Bertenthal BI, Campos JJ, Kermoian R: An epigenetic perspective on the development of self-produced locomotion and its consequences. Psychol Sci 1994;3:140-145.

28 Campos JJ, Anderson DI, Barbu-Roth MA, Hubbard EM, Hertenstein MJ, Witherington D: Travel broadens the mind. Infancy 2000; 1:149-219.
29 Van der Meer AL, Fallet G, Van der Weel FR: Perception of structured optic flow and random visual motion in infants and adults: a high-density EEG study. Exp Brain Res 2008; 186:493-502.

30 Grieve PG, Emerson RG, Fifer WP, Isler JR, Stark RI: Spatial correlation of the infant and adult electroencephalogram. Clin Neurophysiol 2003;114:1594-1608.

31 Webb SJ, Long JD, Nelson CA: A longitudinal investigation of visual event related potentials in the first year of life. Dev Sci 2005; 8:605-616.

32 Coch D, Skendzel W, Grossi G, Neville H: Motion and color processing in school-age children and adults: an ERP study. Dev Sci 2005;8:372-386.

33 Dubois J, Dehaene-Lambertz G, Soarès C, Cointepas Y, Le Bihan D, Hertz-Pannier L: Microstructural correlates of infant functional development: example of the visual pathways. J Neurosci 2008;28:1943-1948.

34 Johnson MH: Functional brain development in infants: elements of an interactive specialization framework. Child Dev 2000;71:7581.

35 Kayed NS, Van der Meer AL: Timing strategies used in defensive blinking to optical collisions in 5- to 7-month-old infants. Infant Behav Dev 2000;23:253-270.

36 Kayed NS, Van der Meer AL: Infants timing strategies to optical collision: a longitudinal study. Infant Behav Dev 2007;30:50-59.

37 Kayed NS, Farstad H, Van der Meer AL: Preterm infants' timing strategies to optical collisions. Early Hum Dev 2008;84:381-388.

38 Jacobs DM, Runeson S, Michaels CF: Learning to visually perceive the relative mass of colliding balls in locally and globally constrained task ecologies. J Exp Psychol Hum Percept Perform 2001;27:1019-1038.

39 Michaels CF, De Vries MM: Higher-order and lower-order variables in the visual perception of relative pulling force. J Exp Psychol Hum Percept Perform 1998;24:526546.

40 Tucker D: Spatial sampling of head electric fields: the geodesic sensor net. Electroencephalogr Clin Neurophysiol 1993;87:287302.

41 Van der Weel FR, Van der Meer AL: Seeing it coming: infants' responses to looming danger. Naturwissenschaften 2009;96:13851391.

42 Ferree TC, Luu P, Russell GS, Tucker DM: Scalp electrode impedance, infection risk, and EEG data quality. Clin Neurophysiol 2001;112:536-544.
43 Picton TW, Bentin S, Berg P, Donchin E, Hillyard SA, Johanson R Jr, et al: Guidelines for using human event-related potentials to study cognition: recording standards and publication criteria. Psychophysiology 2000; 37:127-152.

44 Di Russo F, Matrinez A, Sereno MI, Pitzalis S, Hillyard SA: Cortical sources of the early components of the visual evoked potential. Hum Brain Mapp 2001;15:95-111.

45 Hoechstetter K, Bornfleth H, Weckesser D, Ille N, Berg P, Scherg M: BESA source coherence: a new method to study cortical oscillatory coupling. Brain Topogr 2004;16:233238.

46 Scherg M: Fundamentals of dipole source potential analysis; in Grandori F, Hoke M, Romani GL (eds): Auditory Evoked Magnetic Fields and Electric Potentials: Advances in Audiology Behaviour. Basel, Karger, 1990, pp 40-69.

47 Kayed NS, Van der Meer AL: A longitudinal study of prospective control in catching by full term and preterm infants. Exp Brain Res 2009; 194:245-258

48 Caljouw SR, Van der Kamp J, Savelsbergh GJP: Catching optical information for the regulation of timing. Exp Brain Res 2004; 155:427-438.

49 Jacobs RA: Computational studies of the development of functionally specialized neural models. Trends Cogn Sci 1999;3:31-38.

50 Picton TW, Taylor MJ: Electrophysiological evaluation of human brain development. Dev Neuropsychol 2007;31:249-278.

-51 Geng X, Gouttard S, Sharma A, Gu H, Styner M, Lin W, Gerig G, Gilmore JH: Quantitative tract-based white matter development from birth to age 2 years. Neuroimage 2012;61: 542-557.

52 Lee DN: A theory of visual control of braking based on information about time-to-collision. Perception 1976;5:437-459.

53 Field DT, Wann, JP: Perceiving time to collision activates the sensorimotor cortex. Curr Biol 2005; 15:453-458.

54 Bear MF, Conners BW, Paradiso MA: Neuroscience: Exploring the Brain, ed 3. Baltimore, Lippincott Williams \& Wilkins, 2007.

55 Nunez PL: Electric Fields and the Brain. New York, Oxford University Press, 1981

56 Gilmore RO, Baker TJ, Grobman KH: Stability in young infants' discrimination of optic flow. Dev Psychol 2004;40:259-270. 